\title{
QUASI-STATIC APPROACH OF DAMPING FORCES EXERTED UPON A LONG CYLINDER OSCILLATING IN AN AXIAL FLOW
}

\author{
Lise Divaret * \\ Laboratoire de Mécanique \\ des Structures Industrielles \\ Durables (LaMSID) \\ UMR EDF CNRS CEA 8193 \\ Clamart, France \\ Email: lise.divaret@edf.fr
}

\author{
Julien Berland \\ Laboratoire de Mécanique \\ des Structures Industrielles \\ Durables (LaMSID) \\ UMR EDF CNRS CEA 8193 \\ Clamart, France \\ Email: julien-j.berland@edf.fr
}

\author{
Olivier Doaré \\ Unité de Mécanique (UME) \\ ENSTA ParisTech \\ Palaiseau, France
}

\author{
Hassan Berro \\ Laboratoire de Mécanique \\ des Structures Industrielles \\ Durables (LaMSID) \\ UMR EDF CNRS CEA 8193 \\ Clamart, France \\ Email: hassan.berro@edf.fr
}

\begin{abstract}
This study investigates the flow around a cylinder in a nearaxial flow at a Reynolds number of 27000. Both CFD calculations and experiments are performed. Time-mean values of lift force coefficient are investigated against the inclination of the cylinder in the domain of low inclinations $\left(<15^{\circ}\right)$. Pressure distribution and flow profiles are also measured and extracted from the CFD calculation results for a characteristic inclination $\alpha=5^{\circ}$. Numerical results for force and pressure show fair agreement with experiments for inclination below $5^{\circ}$ and reveal that at low angles, the lift force is proportional to the angle. In the framework of a quasi-static approach, the instantaneous damping force exerted on a cylinder oscillating in axial flow is equivalent to the normal force exerted on a cylinder placed in an oblique flow
\end{abstract}

\section{NOMENCLATURE}

$C_{L} \quad$ Lift coefficient.

$C_{N} \quad$ Normal force coefficient.

$C_{p}$ Pressure coefficient.

D Diameter of the cylinder (m).

$F_{N} \quad$ Normal force per unit length $(\mathrm{N} / \mathrm{m})$.

$F_{D} \quad$ Drag force per unit length $(\mathrm{N} / \mathrm{m})$.

$F_{L} \quad$ Lift force per unit length $(\mathrm{N} / \mathrm{m})$.

$\mathrm{L}$ Length of the cylinder (m).

$p_{\infty} \quad$ Far field pressure $(\mathrm{Pa})$.

Re Reynolds number, based on the cylinder diameter and the incoming flow velocity.

$U_{\infty}$ Incoming flow velocity $(\mathrm{m} / \mathrm{s})$.

$U_{\text {parallel }}$ Longitudinal component of the flow velocity $(\mathrm{m} / \mathrm{s})$.

$x$ Distance to the end of the cylinder along the cylinder $(\mathrm{m})$.

$\alpha$ Inclination of the cylinder.

*Address all correspondence to this author. 
$\rho$ Density of the fluid $\left(\mathrm{kg} / \mathrm{m}^{3}\right)$.

$\theta$ Angular position of the pressure tap.

\section{INTRODUCTION}

A fuel assembly comprises an array of fuel rods maintained by grids and subjected to an axial flow. A description of the forces exerted upon a cylinder oscillating laterally in an axial flow is useful in the nuclear engineering to represent the forces on a fuel assembly during an earthquake. In order to understand physical phenomena involved, the behavior of only one cylinder oscillating in axial flow is investigated.

The study is focused on the damping force, which is the dissipative force component in the direction of the oscillation and orthogonal to the axis of the cylinder. More precisely, the force acting upon a laterally oscillating cylinder can be expanded in a term proportional to the acceleration, which stands for the added mass effect, and in a term proportional to the velocity, which dissipates energy and hence generates damping in harmonic regime. This dissipative force shall be denoted from now on 'damping force' because it is responsible for fluid damping when a cylinder oscillates in axial flow. In the framework of the quasi-steady approach, i.e., if the lateral velocity of the cylinder is small compared to the axial flow and if the oscillation frequency is low compared to the turbulence frequencies, the damping force is identical to the normal force exerted upon a cylinder submitted to a near-axial steady flow. One expects the damping force to depend both on the axial flow and the lateral flow velocities. The analysis of the steady force is then relevant for a further investigation of displacement-induced fluid forces in axial flows.

The normal force for a cylinder oscillating in a fluid at rest has been described by Morison [1,2] as the sum of an added mass force and a drag force. The added mass is a concept [3] which presents the advantage of reducing all the inertial forces to one single coefficient. The Morison expansion can serve as a basis for the description of the forces exerted upon a cylinder oscillating in a axial flow [4]. In the case of a cylinder placed in an oblique flow, Taylor [5], showed that for oblique flow with angles higher than $20^{\circ}$, the axial component of the velocity has no influence. Ersdal \& Faltinsen [6] brings out three different cases: for angles lower than $5^{\circ}$, it is not possible to hold the cross flow principle, for angles between $5^{\circ}$ and $20^{\circ}$, it is possible to hold the cross flow principle but in taking into account the state of the boundary layer and beyond $20^{\circ}$ the cross flow principle can hold.

The objective of this paper is to compare the results of experiments carried out at small angles, for a cylinder in an oblique flow to RANS CFD simulations of the experiments. As the cross flow principle [5] cannot hold to small angle cases, the variation of the normal force with the axial and lateral velocities will be carefully studied. The validity of the RANS simulations will be discussed for the velocity field, the wall pressure distribution and the total lift force exerted by the fluid on the cylinder.

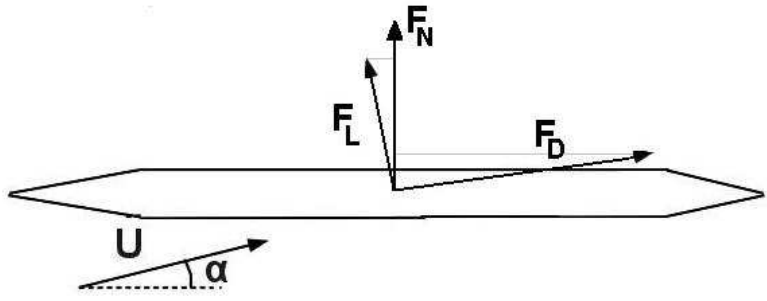

FIGURE 1. VELOCITY IN THE X-DIRECTION, CYLINDER IN NEAR AXIAL FLOW WITH AN ANGLE $\alpha=5^{\circ}$

\section{STATE OF THE ART}

The scientific literature provides very few data about cylinders in near axial flow. Taylor [5] summed up the results of Relf and Powell about the normal force of a cylinder placed in an oblique flow in an air tunnel at angles of inclination between $10^{\circ}$ and $90^{\circ}$. The normal force is the force in a direction normal to the cylinder axis and in the plane formed by the free stream velocity and the cylinder axis (see Fig. 1). In the experiment, the normal force measured is proportional to the square of the lateral component of the velocity. In other words, the normal force measured for an cylinder placed in an oblique flow of velocity $U$ and angle $\alpha$ is the same as the one which would be measured for the same cylinder placed in a cross flow of velocity $U_{\infty} \sin (\alpha)$. This means that the axial component of the flow velocity does not have any influence on the normal force coefficient.

$$
F_{N}=\frac{1}{2} C_{N} \rho_{f} D\left(U_{\infty} \sin \alpha\right)^{2} \quad \text { for } \alpha>20^{\circ}
$$

This result is called the cross flow principle. According to the equation (1), the normal force coefficient $C_{N}$ is around 1.1, which corresponds to the drag of a cylinder in cross flow. This result is however valid only for angles higher than $20^{\circ}$.

Ersdal \& Faltinsen [6] carried out experiments with a cylinder mounted on a towed carriage. The cylinder was towed at a constant velocity and angle $\alpha$ and the normal force was measured. The experiments have been performed by steps of 1 or 2 degrees. Results similar to those of Taylor for angles beyond $20^{\circ}$ where provided. For angles between $5^{\circ}$ and $20^{\circ}$, a transition in the state of the boundary layer was observed: the boundary layer which is laminar for high angles becomes turbulent. In practical terms, it means that the cross flow principle can hold as in the equation (1), but the normal force coefficient is lower in this case than for a laminar boundary layer. Ersdal measured a normal force coefficient $C_{N} \approx 0.8$

For angles lower than $5^{\circ}$, the cross flow principle does not 


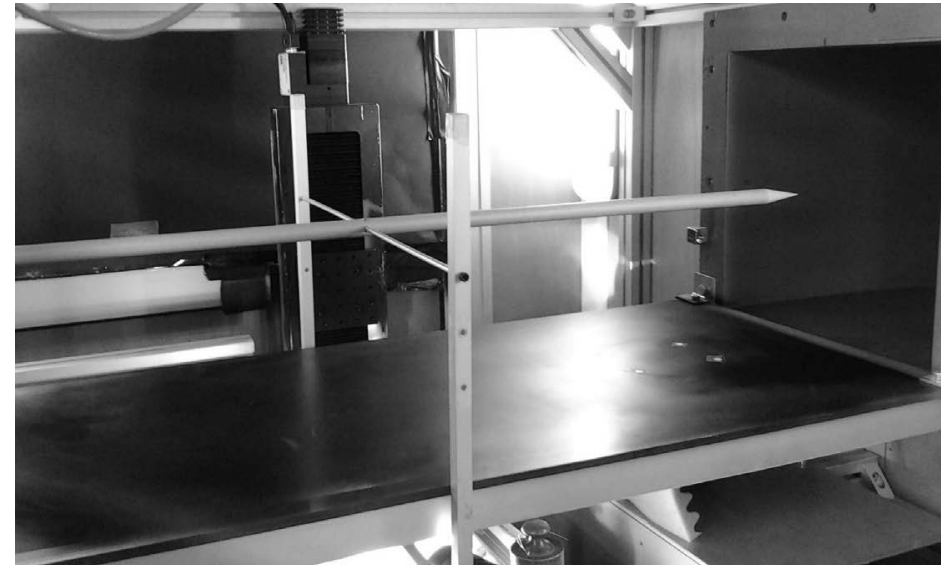

FIGURE 2. EXPERIMENTAL SET UP

hold. Taylor [5] proposed a model based on the friction force which is now traditionnaly used at low angles [7,8]. Ersdal showed that the normal force is proportional to the lateral velocity $U_{\infty} \sin (\alpha)$ (2) and observed that the lift force mostly contributes to the normal force.

$$
F_{N}=\frac{1}{2} C_{N} \rho_{f} D U_{\infty}^{2} \sin \alpha \quad \text { for } \alpha<5^{\circ}
$$

In the present work, the description of the fluid forces is focused on the lift force. For a cylinder in an oblique flow, the lift force $F_{L}$ is the fluid force in a direction orthogonal to free stream flow and in the plane of the flow direction and the cylinder axis (figure 1). The lift force coefficient is defined as the non dimensional lift force by taking the free stream velocity as the reference velocity.

$$
C_{L}=\frac{F_{L}}{\frac{1}{2} \rho_{f} D U_{\infty}^{2}}
$$

\section{EXPERIMENTAL SET UP}

The experiments are performed in an air tunnel as illustrated in Fig. 2. A cylinder is arranged in a near-axial flow with an angle of inclination $\alpha$, varying from $0.5^{\circ}$ (axis almost parallel to the flow) to $15^{\circ}$. The length of the cylinder is $L=1.2 \mathrm{~m}$, its diameter $D=0.02 \mathrm{~m}$ and the ends of the cylinder are coneshaped to prevent flow separation. The incoming flow velocity is measured with a Pitot tube, which exhibits an accuracy of about $0.1 \mathrm{~m} / \mathrm{s}$.

Three types of experiments are performed in the wind tunnel. The goal of the first experiment is to measure the total lift

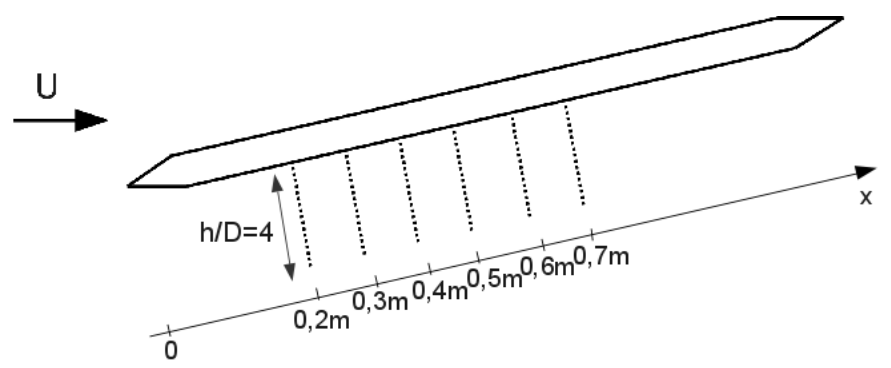

FIGURE 3. POSITION OF THE VELOCITY PROFILES ALONG THE CYLINDER

force exerted on the cylinder. The cylinder is fixed in its middle to a frame with a thin rod. The frame is mounted on a goniometer to control the inclination and the goniometer is fixed to a precision scale to measure the lift force with a precision of $0.05 \mathrm{~g}$. The cylinder force is estimated from the total force by substracting the contribution of the bare frame.

In the second experiment, the velocity profiles along the cylinder are measured with the help of a hot wire probe. Measurements are performed close to the cylinder wall, by varying the distance from 0.4 to $1 \mathrm{~mm}$ by step of $0.1 \mathrm{~mm}$, then from 1 to $5 \mathrm{~mm}$ by step of $1 \mathrm{~mm}$, from 5 to $20 \mathrm{~mm}$ by step of $2.5 \mathrm{~mm}$ and finally from 20 to $80 \mathrm{~mm}$ by step of $10 \mathrm{~mm}$. The probe is arranged at several locations along the cylinder, as shown in Fig. 3: the distance to the end of the cylinder spans from $0.2 \mathrm{~m}$ to $0.7 \mathrm{~m}$ $(x / L=0.17-0.58)$.

The third experiment consists in measuring the pressure at the surface of the cylinder. A pressure tap is arranged at a distance $X=0.46 \mathrm{~m}$ from the end of the cylinder $(X / L=0.38)$. By rotating the cylinder around its axis, the pressure distribution around it can be measured. Results are provided for an angle $\alpha$ equal to $5^{\circ}$, and an angle $\theta$ varying from $0^{\circ}$ to $180^{\circ}$ by steps of $15^{\circ}$. The incoming flow velocity is $20 \mathrm{~m} / \mathrm{s}$, which corresponds to a diameter-based Reynolds number equal to 27000 .

\section{CFD PROCEDURE}

In addition to experiments, CFD simulations have been carried out. The dimensions of the cylinder are the same in the two cases. In order to reduce the calculation time, the fluid domain of the CFD calculations is smaller than the test section of the wind tunnel, but its dimensions are large enough to avoid confinement effects.

Flow motions are solved here using Code Saturne, an EDF in-house open CFD tool [9] based on a collocated finite volume approach. Three dimensional steady RANS simulations are performed with a $k-\omega$ turbulence model. An analysis of the effects of different turbulence models ranges beyond the scope of this paper. For comparison sake, simulations with $k-\varepsilon$ turbulence 


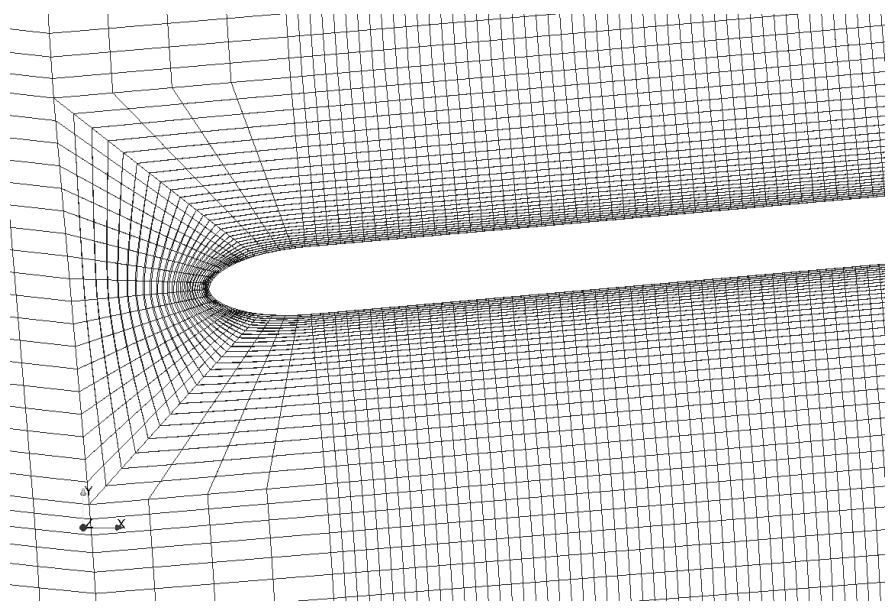

FIGURE 4. MESH, ZOOM ON THE END OF THE CYLINDER

model have been performed but are not shown in the present paper as the results obtained are similar to the ones obtained with the $k-\omega$ turbulence model.

The mesh is built with quadrangle elements only, as shown in Fig. 4. The reference mesh has a number of $1.66 \times 10^{6}$ cells. The cylinder dimensions are the same as in the experiments. The dimensions of the fluid domain are $V=0.92 \mathrm{~m} \times 0.32 \mathrm{~m} \times 1.8 \mathrm{~m}$. The mesh is refined close to the walls of the cylinder $\left(1<y^{+}<\right.$ 30) and a two scale wall law is chosen.

The resolution of the velocity field and pressure is directly linked to the size of the mesh. The pressure distribution around the cylinder is hence determined by steps of $8^{\circ}$, which corresponds to about $1 \mathrm{~mm}$. The same resolution is obtained for the velocity profiles close to the cylinder.

\section{LIFT COEFFICIENT}

The lift coefficient of a cylinder in a steady oblique flow was measured in a previous paper [10], and the main results are shown in Fig. 5, with the notations of Fig. 1. It was shown that the major contribution to the normal force was due to the lift force, and that two different flow regimes occurred, the first exhibiting a linear dependency of the normal force with respect to the angle $\alpha$ for values lower than $5^{\circ}$, and the second exhibiting a quadratic dependency for higher values, consistent with the socalled cross-flow principle, and already observed by Ersdal \& Faltinsen [6].

The results of new experiments, performed with an accurate scale, are reproduced in Fig. 6, and compared to CFD calculations. The experimental results exhibit the same trend as in Fig. 5, i.e., the force is linear for angles lower than $5^{\circ}$, and quadratic beyond. The reasons for this quadratic behavior are related to flow separation effects that shall not be described here, and the discussion is focused in the current study on the linear

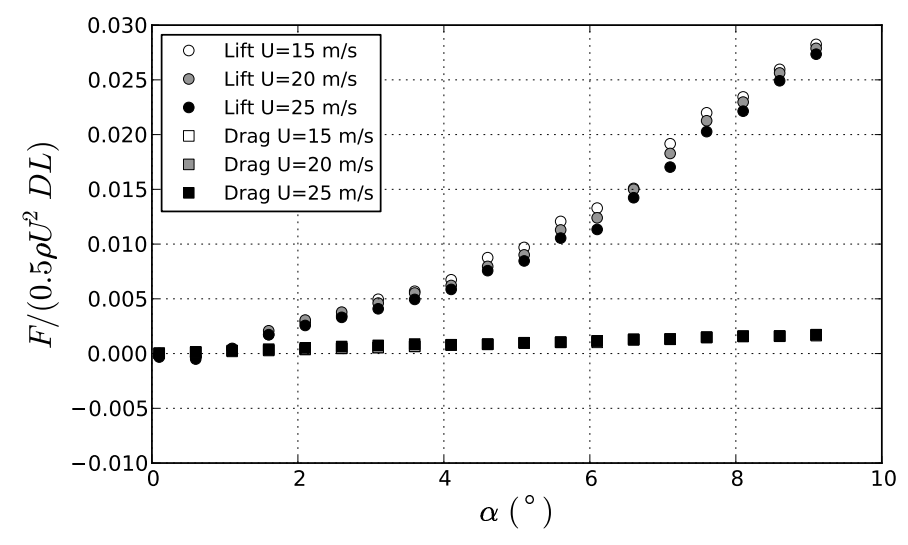

FIGURE 5. EXPERIMENTAL LIFT AND DRAG COMPONENT OF THE NORMAL FORCE VS. INCLINATION ANGLE [10]

TABLE 1. SLOPE OF THE LIFT COEFFICIENT FOR $\alpha<5^{\circ}$

\begin{tabular}{lll}
\hline & Experiments & Simulations \\
\hline$C_{L} / \alpha[\mathrm{rad}]$ & 0.010 & 0.0125 \\
\hline
\end{tabular}

range.

A qualitative agreement is obtained between the CFD calculations and the measurements, as illustrated by the experimental dashed line in Fig. 5, and by the slope estimation in Table 1. Discrepancies appear in the range of angles beyond $5^{\circ}$, but the small angle is correctly predicted by the simulations.

\section{FLOW PATTERN}

The observation of the flow pattern performed with the hot wire reveals that no significant r.m.s. velocity exists except very close to the cylinder, and the RANS simulation exhibits a similar trend, as shown in Fig. 7. It can hence be assessed that no large unsteady structures are generated by the oblique flow at low angles. More specifically, a gradual deficit of velocity is observed during the experiments and predicted by the RANS simulation on the lower side $\left(\theta=180^{\circ}\right)$ (see Figs. 8 and 9), with a thickness varying from 1.0 to $1.5 \mathrm{D}$, whereas it is almost constant on the upper side $\left(\theta=0^{\circ}\right)$. This situation is highly different from crossflow cases $[7,11,12]$, where unsteady vortices are shed along the separation line. This absence of a clear flow separation is a distinct feature of quasi-oblique flow, which does not seem to have been reported up to now.

Some discrepancies are present when comparing the CFD and experimental velocity profiles; the velocity gradient is 


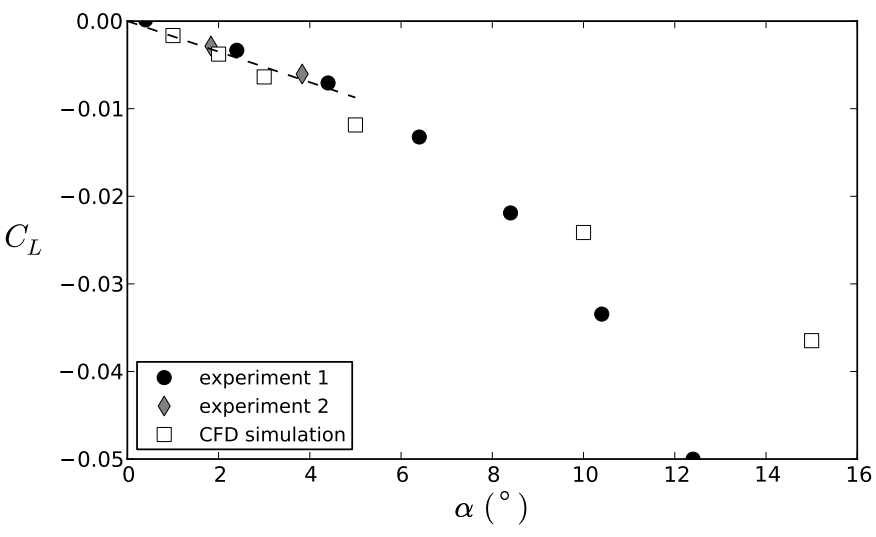

FIGURE 6. LIFT COEFFICIENT VS. INCLINATION ANGLE, EXPERIMENTAL POINTS AND RESULTS OF THE CFD CALCULATIONS

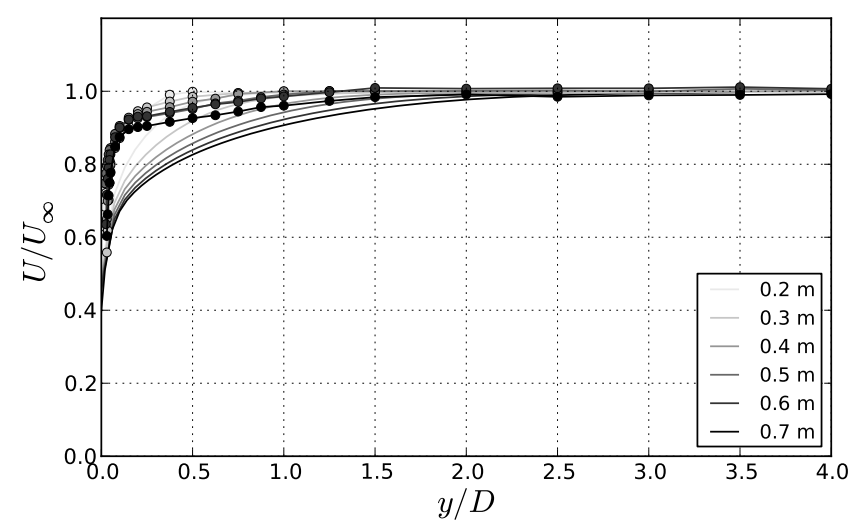

FIGURE 7. VELOCITY PROFILES: • EXPERIMENTS AND CFD SIMULATIONS

smaller in the computations, and the deficit area is larger, which seems to indicate that the RANS approach overestimates diffusion effects close to the cylinder wall. This point shall be discussed at longer extent in the discussion section.

Another feature of the oblique flow is the variation of the thickness of the velocity deficit area along the cylinder, which indicates that the flow pattern is three-dimensional. The consequences with respect to the definition of a lift coefficient have been investigated in the previous section.

One expects the lift force to be related to the velocity difference between the upper side and the lower side, but further work is needed to unambiguously assess this point.

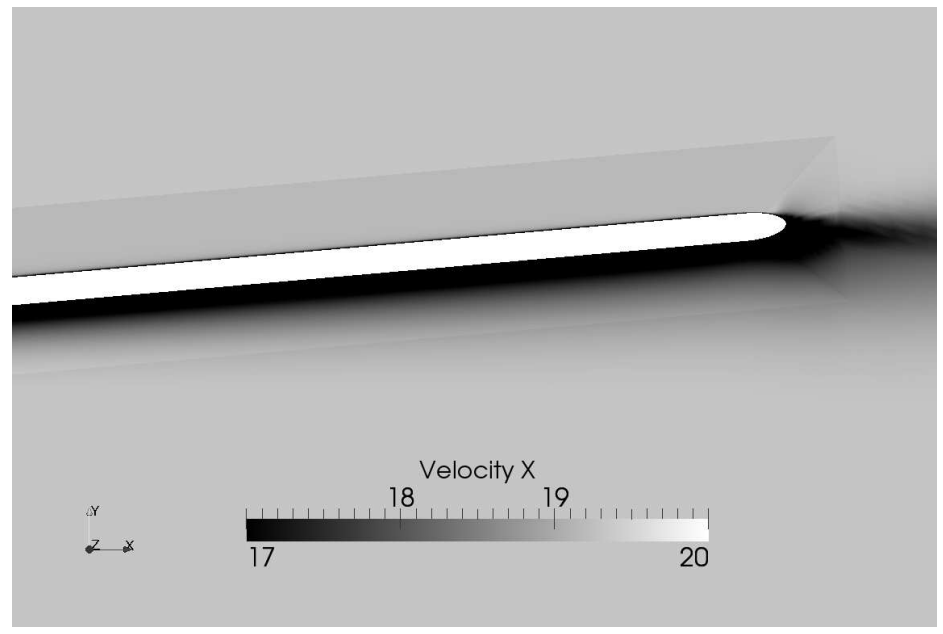

FIGURE 8. VELOCITY IN THE X-DIRECTION, CYLINDER IN NEAR AXIAL FLOW WITH AN ANGLE $\alpha=5^{\circ}$, LONGITUDINAL SECTION

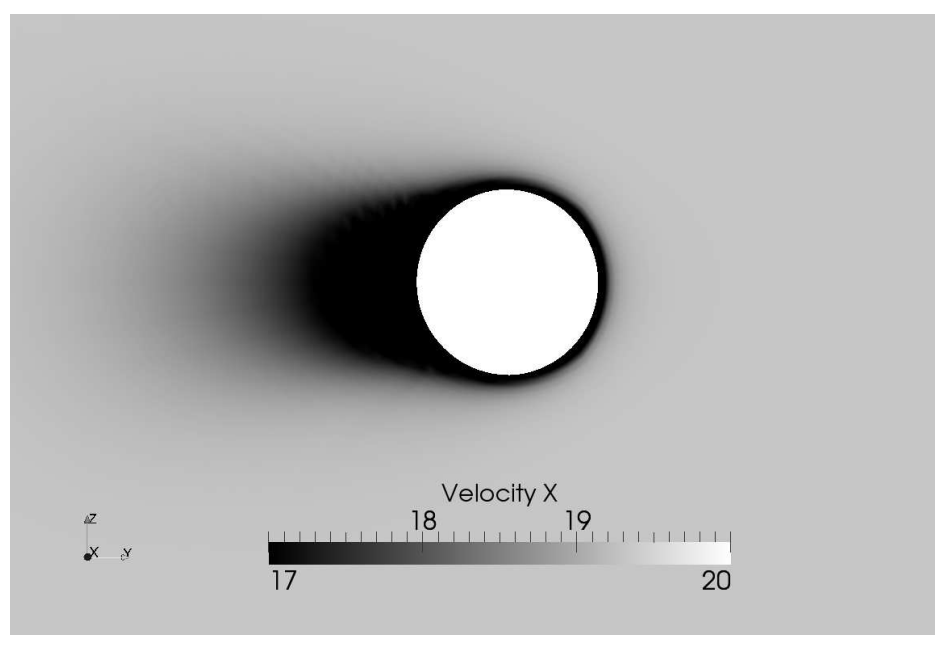

FIGURE 9. VELOCITY IN THE X-DIRECTION, CYLINDER IN NEAR AXIAL FLOW WITH AN ANGLE $\alpha=5^{\circ}$, TRANSVERSE SECTION

\section{PRESSURE DISTRIBUTION ALONG THE CYLINDER WALL.}

The coefficient $C_{p}$ in Eq. (4) is defined as a dimensionless pressure difference, with the far field pressure chosen as reference. The Fig. 10 shows the convention for the angular position of the pressure tap on the instrumented cylinder.

$$
C_{p}=\frac{p-p_{\infty}}{\frac{1}{2} \rho_{f} U_{\infty}^{2}}
$$




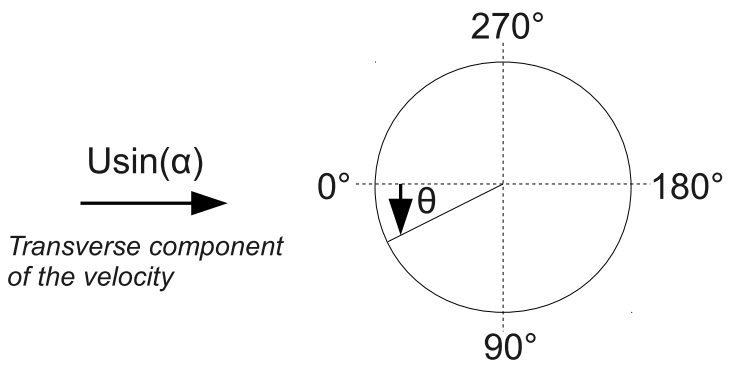

FIGURE 10. ANGULAR POSITION OF THE PRESSURE TAP

The experimental and computed pressure distributions are compared in Fig. 11. At $\theta=0^{\circ}$, the pressure coefficient is maximal but it is strictly lower than 1 , which differs from cross flow pressure distributions where there is a stagnation point and $C_{p}=1$. More precisely, if the velocity were equal to zero at $\theta=0^{\circ}$, a direct application of the Bernoulli equation would lead to $C_{p}=1$. The other way around, as the pressure coefficient is not equal to 1 , one expects a non-vanishing flow velocity parallel to the axis at $\theta=0^{\circ}$, which can be estimated by applying the Bernoulli equation (5).

$$
U_{\infty}^{2}+\frac{p_{\infty}}{\rho_{f}}=U_{\text {parallel }}^{2}+\frac{p_{\theta=0}}{\rho_{f}}
$$

A value of $U_{\text {parallel }}=19.88 \mathrm{~m} \cdot \mathrm{s}^{-1}$ is obtained, very close to the incoming flow velocity equal to $20 \mathrm{~m} / \mathrm{s}$. Further work is needed to accurately describe the flow pattern: whether the flow is deviated around the cylinder or convected along the line $\theta=0$ is not clear, and cannot be easily assessed in the framework of the present study.

Between $\theta=0^{\circ}$ and $\theta=80^{\circ}$, the pressure coefficient gradually decreases, as is the case in cross flow studies where the velocity increases. It reaches its minimal value at $\theta_{\min }=80^{\circ}$, and increases between $\theta=80^{\circ}$ and $\theta=180^{\circ}$, where it is equal to about -0.005 , a negative value which is consistent with the velocity deficit observed in the previous section.

In Fig. 11, a fairly reasonable agreement is obtained between the experimental and the calculated pressure distribution around the cylinder. The values of the pressure at the upper side $\theta=0^{\circ}$, the lower side $\theta=180^{\circ}$ and at $\theta_{\min }$ are close. However, the minimum angle $\theta_{\min }$ is equal to $80^{\circ}$ in the experiments and to $80^{\circ}$ in CFD calculations. Furthermore, the pressure distribution is steeper in the calculation close to $\theta_{\min }$, and flatter at the upper side and the lower side.

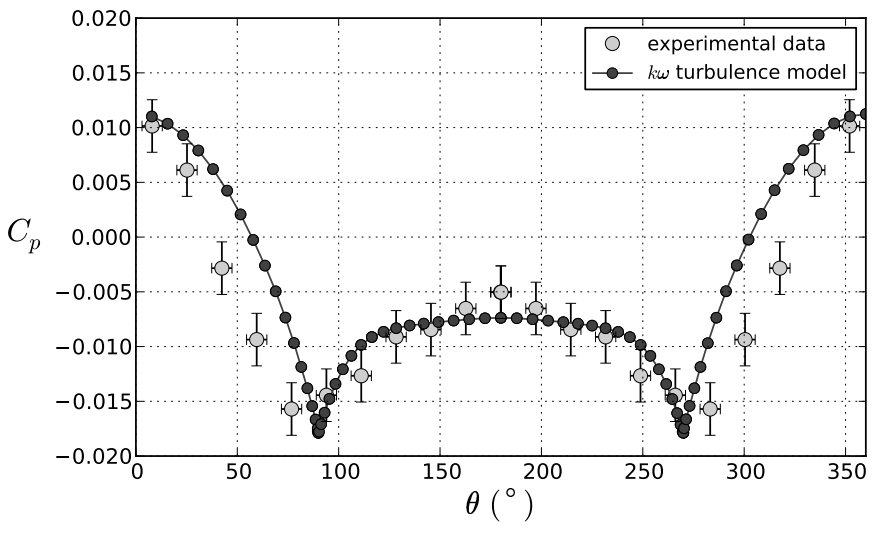

FIGURE 11. MEAN PRESSURE DISTRIBUTION AROUND THE CYLINDER AT AN INCLINATION $\alpha=5^{\circ}$

\section{DISCUSSION \\ Validity of the velocity flow with RANS calculations.}

As pointed out in Fig. 7, even though the main trends of the flow field are predicted by the present RANS simulations, significant discrepancies are still observed for the fine flow features in the vicinity of the cylinder wall.

In particular, the velocity deficit area is larger in RANS calculations compared to those observed in the experiments: it turns out that the boundary layer thickness provided by the simulations is overestimated. For such flow configuration, spatial flow development in the streamwise direction is driven the details of the boundary layer dynamics: a laminar breakdown occurs near the upstream tip of the cylinder and is followed by the emergence of a spatially developing turbulent boundary layer further downstream.

Unfortunately, such flow scenario falls outside the scope of calculations based on RANS turbulence modelling, and fine mechanisms such as the transition to turbulence in a boundary layer are then clearly out reach. Closures for Reynolds-averaged equations are indeed designed and calibrated for fully turbulent steady flows. As a consequence, during a RANS simulation, the turbulence model considers all boundary layers as being fully turbulent. Unwanted extra-dissipation is therefore added and the boundary layer thicknesses are concurrently overestimated.

\section{Validity of the pressure.}

The plot of the lift coefficient as a function of the inclination angle in Fig. 6 highlights an interesting fact: despite the inability of RANS calculations to reproduce the turbulent boundary layer details, it seems that a fair collapse between simulations and experiments is seen for small enough angles $\left(\alpha<5^{\circ}\right)$. One could state that for low angles of attack, the lift coefficient mainly de- 
pends on the main flow features, so that the details close to the solid walls would not have a significant influence. Further investigations are nonetheless clearly required to further assess this assumption.

According to the experiments, for larger angles of inclination $\left(\alpha>5^{\circ}\right)$ flow separation is expected with a loss of linearity between the lift coefficient and the angle $\alpha$. The present simulations thus seem to miss the onset of this new flow regime since the lift force remains a linear function of the angle of inclination over the range investigated $\left(0^{\circ}<\alpha<15^{\circ}\right)$.

The CFD data are however obtained thanks to a steady RANS exhibiting poor capabilities considering the prediction of an unsteady turbulent phenomena such as the flow separation in the wake of a cylinder. In addition, the flow structure is likely to be three-dimensional while the $k-\omega$ turbulence model is isotropic. Though they have not been assessed here, unsteady RANS, second-order closures (i.e. anisotropic) might yield more consistent results.

\section{CONCLUSION}

Experiments and CFD calculations have been carried out to determine the lift force exerted on a cylinder in near-axial flow. The experimental results are consistent with the results obtained by Ersdal \& Faltinsen [6] and with previous results [10], namely, for inclination angles lower than $5^{\circ}$, the lift force appears proportional to the inclination angle. The velocity pattern and the pressure distribution around the cylinder indicate that the lift force is somehow related to a gradual velocity deficit at the lower side, without any clear flow separation.

The CFD computations and the experiments exhibit a reasonable agreement with respect to the minimum and the maximum pressure. The measured velocity deficit is predicted by the computation but its thickness is overestimated. This can be explained by the fact that the RANS modeling is designed for fully turbulent flow, with an isotropic turbulence model.

From a global point of view, the lift forces obtained by RANS CFD calculations are close to the experimental results for inclinations lower than $5^{\circ}$. Despite some modeling inaccuracies close to the wall, the RANS calculations seem able to predict the general trend of the lift force for small angles of inclinations.

Further work is needed to determine the range of validity of the quasi-static approach. The damping forces of a cylinder oscillating in axial flow should be investigated for low oscillation frequencies $(f<U / L)$ and low lateral velocities or low instantaneous angle $\left(\alpha(t)<5^{\circ}\right)$ and then compared to the normal force values predicted with the quasi-static approach.

\section{REFERENCES}

[1] J.R. Morison, M. O. J. J., and Schaaf, S., 1950. "The force exerted by surface waves on piles". Petroleum Transactions, AIME, 189, pp. 149-154.

[2] Sarpkaya, T., 2001. "On the force decompositions of Lighthill and Morison”. Journal of Fluids and Structures, 15, pp. 227-233.

[3] Sarpkaya, T., 2004. "A critical review of the intrinsic nature of vortex-induced vibrations". Journal of Fluids and Structures, 19(4), pp. 389-447.

[4] Sarpkaya, T., 1986. "Force on a circular cylinder in viscous oscillatory flow at low keulegancarpenter numbers". Journal of Fluid Mechanics, 165, pp. 61-71.

[5] Taylor, G., 1952. "Analysis of the swimming of long and narrow animals". Proceedings Royal Society of London, 214, pp. 158-183.

[6] Ersdal, S., and Faltinsen, O., 2006. "Normal forces on cylinders in near-axial flow". Journal of Fluids and Structures, 22(8), pp. 1057-1077.

[7] Blevins, R., 1990. Flow-induced vibration. Van Nostrand Reinhold.

[8] Païdoussis, M., 2004. Fluid-structure interactions: slender structures and axial flow. Fluid-structure interactions. Academic Press.

[9] Archambeau, F., Sakiz, M., and Namane, M., 2004. "Code saturne: a finite volume code for turbulent flows". Int. J. Finite Vol., 1(1), pp. 1-62.

[10] Divaret, L., Cadot, O., Doaré, O., Moussou, P., and Berland, J., 2012. "Normal forces exerted upon a long cylinder oscillating in an axial flow". Proceedings of the 10th International conference on Flow-induced vibration.

[11] Zdravkovich, M., 1997. Flow around circular cylinders, oxford university press, new york ed., Vol. 1.

[12] Païdoussis, M. P., Price, S. J., and de Langre, E., 2011. Fluid-Structure Interactions: Cross-Flow-Induced Instabilities, cambridge university press, cambridge ed. 